\title{
Validação de conteúdo e aparente de check list adaptado para contagem de material cirúrgico
}

\author{
Content validation and apparent check list adapted for surgical material count \\ Validación de contenido y lista de verificación aparente adaptada para el recuento de material \\ quirúrgico
}

Recebido: 23/12/2021 | Revisado: 02/01/2022 | Aceito: 21/01/2022 | Publicado: 22/01/2022

\author{
Antônia Abigail do Nascimento Cavalcante \\ ORCID: https://orcid.org/0000-0001-5172-6088 \\ Universidade Estadual do Ceará, Brasil \\ E-mail: abigail.nascimento123@gmail.com \\ Maria Salete Bessa Jorge \\ ORCID: https://orcid.org/0000-0001-6461-3015 \\ Universidade Estadual do Ceará, Brasil \\ E-mail: maria.salete.jorge@gmail.com
}

\begin{abstract}
Resumo
Validar um checklist adaptado para contagem de material cirúrgico. Trata-se de um estudo metodológico desenvolvido utilizando a validação de conteúdo e aparente onde a revisão integrativa foi utilizada como método de busca que subsidiou a adaptação do checklist proposto e fortaleceu a relevância da pesquisa para a segurança do paciente cirúrgico. Para tanto, participaram sete juízes especialistas para a validação do instrumento do checklist. E o Índice de Validade de Conteúdo (IVC) foi adotado como a taxa de concordância de igual ou superior a 0,80. Os resultados demonstram que o instrumento em análise obteve nota 0,95 na sua avaliação global em relação à validação de conteúdo, e o IVC encontrado foi de 0,97 no que concerne à validação aparente, o que revela a aprovação do instrumento em relação ao conteúdo e aparência. Com isso, percebe-se a necessidade de instrumentos e ações que proporcionem a promoção da segurança do paciente e dos profissionais relacionados ao ambiente cirúrgico, visto que a segurança do paciente é uma dimensão essencial da qualidade do cuidado em saúde. A utilização e entendimento corretos por parte dos profissionais acerca desse instrumento é importante para solidificar a centralidade do cuidado no paciente, propiciando uma comunicação mais eficaz entre os profissionais das equipes multiprofissionais, garantindo assim a prática de uma cirurgia segura.
\end{abstract}

Palavras-chave: Lista de checagem; Segurança do paciente; Validação de dados.

\begin{abstract}
Validate an adapted checklist for counting surgical material. This is a methodological study developed using face and content validation, and the integrative review was used as a search method that supported the adaptation of the proposed checklist and strengthened the relevance of the research for the safety of surgical patients. For this purpose, seven expert judges participated in the validation of the checklist instrument. And the Content Validity Index (CVI) was adopted as an agreement rate equal to or greater than 0.80 . The results show that the instrument under analysis obtained a score of 0.95 in its overall assessment in relation to content validation, and the CVI found was 0.97 in relation to apparent validation, which reveals the approval of the instrument in relation to the content and appearance. Thus, the need for instruments and actions that promote the safety of patients and professionals related to the surgical environment is perceived, as patient safety is an essential dimension of the quality of health care. The correct use and understanding by professionals of this instruments important to solidify the centrality of patient care, providing more effective communication between professionals in the multidisciplinary teams, thus ensuring the practice of safe surgery.
\end{abstract}

Keywords: Checklist; Patient safety; Data validation.

\section{Resumen}

Validar una lista de verificación adaptada para contar material quirúrgico. Se trata de un estudio metodológico desarrollado mediante validación facial y de contenido, y se utilizó la revisión integradora como método de búsqueda que apoyó la adecuación del checklist propuesto y fortaleció la relevancia de la investigación para la seguridad de los pacientes quirúrgicos. Para ello, siete jueces expertos participaron en la validación del instrumento de checklist. Y se adoptó el Índice de Validez de Contenido (IVC) como tasa de acuerdo igual o superior a 0,80. Los resultados muestran que el instrumento analizado obtuvo una puntuación de 0,95 en su valoración global en relación a la validación de contenido, y el IVC encontrado fue de 0,97 en relación a la validación aparente, lo que revela la aprobación del instrumento en relación al contenido y apariencia. Así, se percibe la necesidad de instrumentos y acciones que 
promuevan la seguridad de los pacientes y profesionales relacionados con el entorno quirúrgico, ya que la seguridad del paciente es una dimensión esencial de la calidad de la atención en salud. El correcto uso y comprensión por parte de los profesionales de este instrumento es importante para solidificar la centralidad de la atención al paciente, proporcionando una comunicación más efectiva entre los profesionales de los equipos multidisciplinares, garantizando así la práctica de una cirugía segura.

Palabras clave: Lista de verificación; Seguridad del paciente; Validación de datos.

\section{Introdução}

O Programa "Cirurgias Seguras Salvam Vidas", proposto com detalhes em 2008 pela OMS, contempla dez objetivos essenciais para a realização de uma cirurgia segura, que englobam a conferência de que o paciente e o local correto estão sendo operados, que a equipe está preparada para impedir danos que possam vir a ocorrer com relação a administração de anestésicos, manejo de via aérea, perda sanguínea, infecção, retenção de compressas e/ou instrumentais e encaminhamento de espécimes cirúrgicos e que a comunicação efetiva deverá ser implementada (WHO,2009).

O programa apresenta para execução desses objetivos listas de verificação ou checklists que auxiliam a conferência de elementos essenciais relativos à segurança do paciente durante a realização de um procedimento cirúrgico. $\mathrm{O}$ checklist proposto pela OMS é dividido em três tópicos: 1. Identificação (sign in); 2. Confirmação (time out) e 3. Registro (sign out) (WHO,2009).

A implantação e aplicação do checklist de cirurgia segura em sala operatória, apesar de ter baixo custo e alcançar resultados considerados satisfatórios, não é algo simples, como pode parecer à primeira vista. Envolve múltiplos aspectos desde o conhecimento do condutor até a aplicação em equipe, além de aspectos estruturais e organizacionais, sendo, portanto, considerado uma tecnologia de moderada dificuldade de implantação (Ribeiro et al., 2017).

$\mathrm{Na}$ assistência cirúrgica, o uso de checklist tem sido alvo de estudos sobre sua aplicação, nas diferentes fases no cotidiano de trabalho, considerando o fato de ser uma ferramenta que permite a checagem de itens importantes que poderiam ser esquecidos pela equipe. No Brasil, há escassez de estudos que mostrem métodos de trabalho no processo de implantação e de preenchimento adequado do instrumento bem como sua importância para a redução de incidentes (Ribeiro et al., 2017).

O checklist pode ser empregado em qualquer hospital, independente do seu porte e condição financeira (Feraz, 2009), devendo ser adaptado de acordo com a realidade do hospital e utilizada por todos os profissionais da saúde envolvidos no período perioperatório, contudo deve ser conduzido por uma única pessoa.

Existem evidências de que a adesão ao checklist tem sido pouco explorada, especialmente em países em desenvolvimento e, particularmente, na América Latina. Porém, a análise da utilização dessa ferramenta permite identificar problemas e fatores associados ao seu efetivo uso para possibilitar a incorporação da tecnologia, visando aos resultados positivos para a segurança do paciente cirúrgico (Freitas et al., 2014).

Baseado nos dados apresentados pela Agência Nacional de Vigilância Sanitária (ANVISA) (Brasil, 2017) foram notificados 3.771 Never Events (eventos que nunca deveriam ocorrer em serviços de saúde), sendo 66 (1,7\%) decorrentes de Retenção não Intencional de Objetos no paciente após Cirurgia e quanto às notificações de óbitos no período de janeiro de 2014 a julho de 2017, 46 (6\%) ocorreram devido às falhas durante a realização de procedimentos cirúrgicos.

De acordo com o relatório nacional de Incidentes relacionados à assistência à Saúde, notificados pelos núcleos de segurança do paciente (NSP) dos serviços de saúde, no período de janeiro de 2014 a julho de 2017, dos 134.501 incidentes notificados, 574 corresponderam às falhas durante a realização de procedimentos cirúrgicos (Brasil, 2017).

Sabe-se que é essencial que os profissionais de saúde conheçam o protocolo de Cirurgia Segura, o implementem e vivenciem a construção de uma cultura de segurança visando uma melhor comunicação entre a própria equipe e paciente e assim, evitem eventos adversos. Pensando nisso, devem dispor de estratégias para implementar essa nova tecnologia para o cuidado seguro (Criado et al., 2017). Com isso, o presente estudo objetivou validar um checklist adaptado para contagem de material cirúrgico. 


\section{Metodologia}

Trata-se de um estudo metodológico desenvolvido para validação de um checklist para contagem de material cirúrgico, utilizando a validação aparente e conteúdo. Uma vez que, considera-se a validade como um critério importante para a avaliação de um instrumento quantitativo, que indica em que grau o instrumento mede aquilo que supostamente deve medir (Polit $\&$ Beck, 2011).

A validade apresenta uma série de aspectos, e um deles é a validade aparente. Esta indica se um instrumento parece estar medindo o constructo apropriado, especialmente de acordo com aqueles que vão usar o instrumento. Já a validação de conteúdo, indica em que medida o instrumento possui uma amostra apropriada de itens para medir o constructo específico e cobrir adequadamente seu domínio (Polit \& Beck, 2011).

Para o alcance dos resultados da pesquisa e com o intuito de subsidiar a validação proposta, a revisão integrativa foi utilizada como método de busca pelo entendimento relacionado à prática de contagem cirúrgica em outras instituições, como também, da existência de checklists específicos para este fim. Esta revisão subsidiou a adaptação do checklist proposto e fortaleceu a relevância da pesquisa para a segurança do paciente cirúrgico. Assim, o material a ser validado foi adaptado conforme recomendações da literatura, protocolos vigentes e das necessidades relatadas por profissionais de enfermagem atuantes em centros cirúrgicos, que se deu mediante contribuição dos enfermeiros para que houvesse a adaptação do checklist para contagem de material cirúrgico existente nos centros cirúrgicos estudados, perfazendo-se assim, a importância dele como ferramenta de segurança para o paciente cirúrgico.

Para a validação, é necessária a participação de juízes especialistas na área temática da pesquisa, nesse sentido, recomenda-se um número de seis a 20 juízes e diante desta recomendação, adotou-se sete juízes especialistas para a validação do checklist proposto (Pasquali, 1998).

A escolha dos juízes foi norteada pelo sistema de pontuação de Fehring (1994), sendo classificado aqueles que atingiram pontuação mínima de cinco pontos, conforme apresentamos abaixo:

Quadro 1 - Critérios de seleção para juízes de Fehring, 1994, adaptado por Cavalcante (2019), Fortaleza, CE, Brasil, 2018.

\begin{tabular}{|c|c|c|c|}
\hline Critérios Fehring (1994) & Pontos & Critérios adaptados (2019) & $\begin{array}{c}\text { Pontos } \\
\text { adaptados }\end{array}$ \\
\hline Mestre em enfermagem & 2 & Mestre em enfermagem & 2 \\
\hline $\begin{array}{c}\text { Mestre em enfermagem - } \\
\text { dissertação com conteúdo relevante } \\
\text { dentro da área clínica. }\end{array}$ & 2 & $\begin{array}{c}\text { Mestre em enfermagem - dissertação com conteúdo } \\
\text { relacionado a temática da pesquisa (assistência } \\
\text { cirúrgica e segurança do paciente) }\end{array}$ & 2 \\
\hline $\begin{array}{c}\text { Pesquisa (com publicação) na área } \\
\text { de diagnósticos. }\end{array}$ & 2 & Pesquisa (com publicação) na área de diagnósticos. & 0 \\
\hline $\begin{array}{c}\text { Artigo publicado na área de } \\
\text { diagnósticos em um periódico de } \\
\text { referência. }\end{array}$ & 1 & $\begin{array}{c}\text { Artigo e/ou trabalhos publicado/apresentados na área } \\
\text { da pesquisa. (assistência cirúrgica e segurança do } \\
\text { paciente) }\end{array}$ & 1 \\
\hline $\begin{array}{c}\text { Doutorado em Enfermagem sobre } \\
\text { diagnósticos. }\end{array}$ & 1 & $\begin{array}{c}\text { Doutorado em enfermagem - tese com conteúdo } \\
\text { relacionado a temática da pesquisa. (assistência } \\
\text { cirúrgica e segurança do paciente) }\end{array}$ & 2 \\
\hline $\begin{array}{c}\text { Prática clínica de, pelo menos, um } \\
\text { ano de duração na área de } \\
\text { enfermagem em clínica médica. }\end{array}$ & 2 & $\begin{array}{c}\text { Atuação com no mínimo um ano na prática em centro } \\
\text { cirúrgico e/ou na docência na área da pesquisa. }\end{array}$ & 3 \\
\hline $\begin{array}{c}\text { Certificado na área de clínica } \\
\text { médica com comprovada prática. }\end{array}$ & 2 & $\begin{array}{c}\text { Certificado de Especialista em enfermagem cirúrgica- } \\
\text { TCC com conteúdo relacionado a pesquisa } \\
\text { (assistência cirúrgica e segurança do paciente) }\end{array}$ & 2 \\
\hline Pontuação Máxima & 12 & Pontuação Máxima & 12 \\
\hline
\end{tabular}

Fonte: Adaptado de Fehring (1994).

Conforme critérios estabelecidos, iniciou-se a seleção dos juízes, inicialmente através da plataforma lattes, no intuito 
de selecionar os currículos de acordo com a temática do estudo. Sendo assim, foram encontrados 77 currículos, dos quais foram selecionados sete juízes especialistas para a validação do instrumento do checklist.

Após seleção, foi encaminhado carta convite por e-mail, convidando-os a participarem da pesquisa, bem como o Termo de Consentimento livre e Esclarecido (TCLE), acompanhado do instrumento para notas e concordâncias utilizando a escala Likert e do checklist adaptado. Tal etapa aconteceu em meados de 2019 de forma virtual, estando os pesquisadores residindo na cidade de Fortaleza, Ceará, Brasil.

O processo de validação pelos juízes ocorreu mediante aplicação da Escala de Likert, constando como opções de respostas aos itens as seguintes possibilidades: $1=$ Concordo; $2=$ Discordo totalmente; $3=$ Nem concordo nem discordo; $4=$ Discordo e 5= Discordo totalmente.

A escala de verificação de Likert consiste em tomar um construto e desenvolver um conjunto de afirmações relacionadas à sua definição, para as quais os respondentes emitirão seu grau de concordância. A indicação corrente é de que, uma escala com número ímpar de pontos facilita a resposta por causa do ponto intermediário, que seria um nível neutro entre concordância e discordância, assim, foi utilizado cinco pontos na escala proposta para a pesquisa (Silva \& Costa, 2014).

As respostas obtidas foram avaliadas a partir do grau de concordância dos especialistas através do cálculo do Índice de Validade de Conteúdo - IVC, medido por meio da seguinte proposição:

$$
\mathrm{IVC}=\frac{\text { número de respostas } 4 \text { ou } 5}{\text { número total de respostas }}
$$

Para Alexandre e Coluci (2011), o IVC "compreende um método muito utilizado na área de saúde e permite inicialmente analisar cada item individualmente e depois o instrumento como um todo". Este método emprega uma escala tipo Likert com pontuação de um a cinco.

Sugere-se uma concordância mínima de 0,80 , porém, para atingir a excelência, recomenda-se de 0,90 ou mais (Alexandre \& Coluci, 2011, Polit \& Beck, 2011). Assim, o IVC adotado nesta pesquisa foi uma taxa de concordância de igual ou superior a 0,80 .

\section{Validação aparente}

O instrumento de checklist encontra-se apropriado para utilização, uma vez que o IVC encontrado foi de 0,97, conforme a avaliação dos os juízes participantes do estudo no que concerne à validação aparente. Em relação à validação aparente do checklist, a mesma foi baseada nos itens: clareza da linguagem, organização, aparência e relevância do checklist. 
Quadro 2 - Validação aparente: parecer de avaliação dos juízes especialistas.

\begin{tabular}{|c|c|c|c|c|c|c|c|c|c|}
\hline Dimensão & Aspecto & $\begin{array}{c}\text { JUIZ } \\
1\end{array}$ & $\begin{array}{c}\text { JUIZ } \\
2\end{array}$ & $\begin{array}{c}\text { JUIZ } \\
\mathbf{3}\end{array}$ & $\begin{array}{c}\text { JUIZ } \\
4\end{array}$ & $\begin{array}{c}\text { JUIZ } \\
\mathbf{5}\end{array}$ & $\begin{array}{c}\text { JUIZ } \\
6\end{array}$ & JUIZ 7 & IVC\% \\
\hline \multirow{4}{*}{$\begin{array}{l}\text { Clareza da } \\
\text { linguagem }\end{array}$} & A escrita está clara & 4 & 4 & 4 & 4 & 4 & 4 & 4 & 1 \\
\hline & O vocabulário está apropriado & 4 & 4 & 4 & 4 & 4 & 4 & 4 & 1 \\
\hline & $\begin{array}{l}\text { Os itens estão adequados a proposta do } \\
\text { checklist }\end{array}$ & 4 & 4 & 3 & 4 & 4 & 4 & 4 & 1 \\
\hline & O conteúdo está compreensível & 4 & 4 & 3 & 4 & 4 & 4 & 4 & 1 \\
\hline IVC & & & & & & & & & 1 \\
\hline \multirow[t]{4}{*}{ Organização } & O checklist está bem estruturado & 4 & 4 & 4 & 4 & 4 & 4 & 4 & 1 \\
\hline & A sequência dos itens está adequada & 4 & 4 & 4 & 4 & 4 & 4 & 4 & 1 \\
\hline & $\begin{array}{l}\text { Os espaços para preenchimento estão } \\
\text { adequados }\end{array}$ & 4 & 3 & 3 & 4 & 4 & 4 & 4 & 1 \\
\hline & $\begin{array}{l}\text { Os itens estão organizados conforme } \\
\text { recomendação do protocolo de cirurgia } \\
\text { segura }\end{array}$ & 4 & 4 & 3 & 4 & 4 & 4 & 4 & 1 \\
\hline IVC & & & & & & & & & 1 \\
\hline \multirow[t]{3}{*}{ Aparência } & As sessões estão organizadas & 4 & 4 & 3 & 4 & 4 & 4 & 4 & 1 \\
\hline & $\begin{array}{l}\text { A aparência contribui para fácil } \\
\text { compreensão do instrumento }\end{array}$ & 4 & 4 & 3 & 4 & 4 & 4 & 4 & 1 \\
\hline & $\begin{array}{l}\text { A forma da distribuição dos itens, a } \\
\text { fonte e os espaços estão adequado }\end{array}$ & 4 & 4 & 3 & 4 & 4 & 4 & 4 & 1 \\
\hline IVC & & & & & & & & & 1 \\
\hline \multirow{4}{*}{ Relevância } & $\begin{array}{l}\text { Aborda critérios necessários para a } \\
\text { prevenção da retenção de item na } \\
\text { cavidade cirúrgica }\end{array}$ & 4 & 4 & 2 & 4 & 4 & 4 & 4 & 0,85 \\
\hline & $\begin{array}{l}\text { Consegue mensurar o indicador de } \\
\text { segurança na prevenção de retenção de } \\
\text { itens na cavidade }\end{array}$ & 4 & 4 & 2 & 4 & 3 & 4 & 4 & 0,85 \\
\hline & $\begin{array}{l}\text { Adequado para ser utilizado por } \\
\text { qualquer profissional da equipe }\end{array}$ & 4 & 4 & 2 & 4 & 4 & 4 & 4 & 0,85 \\
\hline & $\begin{array}{l}\text { O checklist atende às recomendações do } \\
\text { protocolo de cirurgia segura da OMS }\end{array}$ & 4 & 4 & 3 & 4 & 4 & 4 & 4 & 1 \\
\hline IVC & & & & & & & & & $\mathbf{0 , 8 9}$ \\
\hline IVC Total & & & & & & & & & 0,97 \\
\hline
\end{tabular}

Fonte: Autores.

Os itens foram separados conforme nota dada pelos juízes em: (1) Discordo, (2) Discordo totalmente, (3) Concordo (4) Concordo totalmente. Identificou-se em cada item a quantidade de juízes que atribuíram notas 3 e 4, em seguida, dividiu-se pelo número total de respostas, ao final os valores foram multiplicados por 100 a fim de obter a porcentagem. Os itens que não receberam concordância acima de $80 \%$ seriam reorganizados conforme sugestão do juiz especialista, porém, não houve nenhuma concordância passível de reorganização.

Por fim, salienta-se que a presente pesquisa foi submetida ao Comitê de Ética em Pesquisa do Instituto de saúde e gestão hospitalar - ISGH recebendo CAAE: 08107919.5.0000.5684. Para todas as etapas da submissão, foram atendidos os princípios éticos da Resolução 466/2012 do Conselho Nacional de Saúde (Brasil, 2012).

\section{Resultados}

\section{Caracterização dos participantes}

Essa fase da pesquisa foi realizada após ajuste do checklist, que se deu mediante contribuições apreendidas dos profissionais e as recomendações dos protocolos e diretrizes vigentes.

Destaca-se que os sete juízes selecionados são enfermeiros, com predomínio do sexo feminino $(71,4 \%)$. Em relação à formação quatro são especialistas $(57,1 \%)$ e três são mestres $(42,9 \%)$. O tempo de atuação em centro cirúrgico variou de quatro a 11 anos, e em relação a cidade de atuação, dois são de Campinas - São Paulo, um de Juazeiro do Norte - Ceará, e quatro de 
Sobral - Ceará.

Em relação à pontuação da caracterização dos juízes, constatou-se que todos os juízes atenderam aos critérios estabelecidos, que era atingir pontuação mínima de cinco pontos. Desta forma, após análise dos currículos dos juízes constatouse que dois atingiram oito (08) pontos, um atingiu sete (07) pontos, dois atingiram seis (06) pontos e dois atingiram cinco (05) pontos.

\section{Validação de Conteúdo}

Os resultados revelam que o instrumento em análise obteve nota 0,95 na sua avaliação global em relação à validação de conteúdo, sendo os itens que compõem o instrumento avaliados com as seguintes pontuações, agulha de sutura ( 0,95$)$, algodão $(0,95)$, compressas $(0,95)$, gaze $(0,95)$, gaze laparoscópica $(0,95)$, dissector $(0,95)$ e instrumental $(0,95)$, sendo que todos os itens foram avaliados considerando os momentos pré - incisão, pré - sutura e pós término cirúrgico.

Com isso, destaca-se que o IVC variou de 0 a 1 , sendo considerado validado, uma vez que se o checklist apresentasse valor de IVC Global maior ou igual a 0,80, este seria considerado validado. Como nenhum dos itens obteve percentual abaixo de $80 \%$ de concordância, não se fez necessário um processo de reformulação de nenhum item. O Quadro 1 a seguir revela as pontuações atribuídas a cada item. 
Quadro 3 - Validação de conteúdo: parecer de avaliação dos juízes especialistas.

\begin{tabular}{|c|c|c|c|c|c|c|c|c|c|}
\hline Material & $\begin{array}{c}\text { Fases da } \\
\text { Contagem }\end{array}$ & Juiz 1 & Juiz 2 & Juiz 3 & Juiz 4 & Juiz 5 & Juiz 6 & Juiz 7 & IVC\% \\
\hline \multirow[t]{3}{*}{ Agulha de Sutura } & Pré-Incisão & 5 & 5 & 5 & 5 & 5 & 5 & 5 & 1 \\
\hline & Pré-Sutura & 5 & 5 & 5 & 5 & 5 & 5 & 4 & 1 \\
\hline & $\begin{array}{c}\text { Após Término } \\
\text { Cirúrgico }\end{array}$ & 5 & 5 & 3 & 5 & 5 & 5 & 4 & 0,85 \\
\hline IVC & & & & & & & & & 0,95 \\
\hline \multirow[t]{3}{*}{ Algodão } & Pré-Incisão & 5 & 5 & 5 & 5 & 5 & 5 & 4 & 1 \\
\hline & Pré-Sutura & 5 & 5 & 5 & 5 & 5 & 5 & 4 & 1 \\
\hline & $\begin{array}{c}\text { Após Término } \\
\text { Cirúrgico }\end{array}$ & 5 & 5 & 3 & 5 & 5 & 5 & 4 & 0,85 \\
\hline IVC & & & & & & & & & $\mathbf{0 , 9 5}$ \\
\hline \multirow[t]{3}{*}{ Compressas } & Pré-Incisão & 5 & 5 & 5 & 5 & 5 & 5 & 4 & 1 \\
\hline & Pré-Sutura & 5 & 5 & 5 & 5 & 5 & 5 & 4 & 1 \\
\hline & $\begin{array}{c}\text { Após Término } \\
\text { Cirúrgico }\end{array}$ & 5 & 5 & 3 & 5 & 5 & 5 & 4 & 0,85 \\
\hline IVC & & & & & & & & & 0,95 \\
\hline \multirow[t]{3}{*}{ Gaze** $^{* *}$} & Pré-Incisão & 5 & 5 & 5 & 5 & 5 & 5 & 4 & 1 \\
\hline & Pré-Sutura & 5 & 5 & 5 & 5 & 5 & 5 & 4 & 1 \\
\hline & $\begin{array}{l}\text { Após Término } \\
\text { Cirúrgico }\end{array}$ & 5 & 5 & 3 & 5 & 5 & 5 & 4 & 0,85 \\
\hline IVC & & & & & & & & & 0,95 \\
\hline \multirow{3}{*}{$\begin{array}{c}\text { Gaze } \\
\text { Laparoscópica** }\end{array}$} & Pré-Incisão & 5 & 5 & 5 & 5 & 5 & 5 & 4 & 1 \\
\hline & Pré-Sutura & 5 & 5 & 5 & 5 & 5 & 5 & 4 & 1 \\
\hline & $\begin{array}{c}\text { Após Término } \\
\text { Cirúrgico }\end{array}$ & 5 & 5 & 3 & 5 & 5 & 5 & 4 & 0,85 \\
\hline IVC & & & & & & & & & 0,95 \\
\hline \multirow[t]{3}{*}{ Dissector } & Pré-Incisão & 5 & 5 & 5 & 5 & 5 & 5 & 4 & 1 \\
\hline & Pré-Sutura & 5 & 5 & 5 & 5 & 5 & 5 & 4 & 1 \\
\hline & $\begin{array}{c}\text { Após Término } \\
\text { Cirúrgico }\end{array}$ & 5 & 5 & 3 & 5 & 5 & 5 & 4 & 0,85 \\
\hline IVC & & & & & & & & & 0,95 \\
\hline \multirow[t]{3}{*}{ Instrumental } & Pré-Incisão & 5 & 5 & 5 & 5 & 5 & 5 & 4 & 1 \\
\hline & Pré-Sutura & 5 & 5 & 5 & 5 & 5 & 5 & 4 & 1 \\
\hline & $\begin{array}{c}\text { Após Término } \\
\text { Cirúrgico }\end{array}$ & 5 & 5 & 3 & 5 & 5 & 5 & 4 & 0,85 \\
\hline IVC & & & & & & & & & 0,95 \\
\hline IVC Total & & & & & & & & & 0,95 \\
\hline
\end{tabular}

Fonte: Autores.

\section{Discussão}

Destaca-se que as modificações realizadas no instrumento foram pertinentes e emergiram a partir das contribuições de profissionais atuantes em três hospitais de um município do interior do estado do Ceará e que contou com a participação de juízes especialistas que consideram o checklist final válido quanto a conteúdo e aparência, pois obteve valores de 0,95 e 0,97, respectivamente.

Assim, semelhante ao presente estudo, o trabalho desenvolvido por Alpendre et al. (2017), as solicitações dos especialistas referiram-se mais à forma de apresentação dos itens do que propriamente ao conteúdo do instrumento. De acordo com o autor, infere-se que a estrutura dos itens do manuscrito está relacionada às necessidades de verificação da segurança cirúrgica, assim, após as alterações, o instrumento foi submetido à segunda rodada de avaliação e todas as questões avaliadas pelos especialistas atingiram concordância $\geq 88 \%$ e ranking médio $\geq 1,38$.

O estudo de Ribeiro et al. (2017) sugere que apenas a inserção da ferramenta no processo de trabalho não assegura a qualidade das práticas, sendo necessário investir na construção de uma cultura de segurança organizacional embasada por 
planejamento, estratégias e avaliação. A ausência de preenchimento de alguns itens indica orientação inadequada sobre o instrumento e sua finalidade, fragilidades na interação e comunicação entre os profissionais envolvidos e reduzida valorização da ferramenta. São básicas as intervenções educacionais e de sensibilização contínuas para a adesão ao checklist visando à segurança cirúrgica dos pacientes.

Nesse sentido, apreende-se que as constantes e intensas mudanças nos dias atuais, nos quais é crescente e cada vez mais acelerada a inovação tecnológica, tem-se à disposição dos profissionais e usuários os mais diversos tipos de tecnologias, tais como: tecnologias educacionais, gerenciais e assistenciais (Sewell et al., 2011). Dessa forma, é importante ter postura crítica e reflexiva sobre a utilização da tecnologia, buscando adequação às necessidades de saúde como um todo.

Com isso, são necessários instrumentos e ações que proporcionem a promoção da segurança do paciente e dos profissionais relacionados ao ambiente cirúrgico (Ribeiro et al., 2017), uma vez que a segurança do paciente é uma dimensão essencial da qualidade do cuidado em saúde, assumindo papel de destaque em centro cirúrgico por se tratar de um ambiente técnico, de grande diferenciação e com uma estrutura específica que impacta em seus resultados (Valido, 2011).

Contudo, existem alguns entraves nesse processo como, principalmente, a resistência dos profissionais médicos, provavelmente resultado do processo de implantação que não os envolveu desde o início, o que levou a valores abaixo da expectativa em todos os anos do estudo (Ribeiro et al., 2017). Em trabalhos desenvolvidos no Brasil, Espanha, Inglaterra e Canadá, os valores de adesão ao preenchimento de seus instrumentos atingiram 60,5\%, 83,3\%, 96,9\% e 92\%, respectivamente (Freitas et al., 2014; Sault Area Hospital, 2015; Sewell et al., 2011; Soria-Aledo et al., 2012).

Nesse mesmo sentido, o presente estudo contou apenas com a colaboração e participação de profissionais de Enfermagem (técnicos e enfermeiros), no intuito de sanar essas barreiras, uma vez que estes na maioria das vezes são os responsáveis pelo preenchimento do instrumento.

Apesar de ser uma ferramenta extremamente eficaz para diminuir os eventos adversos, observado a partir de sustentação literária, entretanto sua implantação efetiva é desafiadora nos hospitais (Ferreira, Ribeiro, Mendonça, \& Amaro, 2019). A correta utilização e entendimento por parte dos profissionais acerca desse instrumento é importante para solidificar a centralidade do cuidado no paciente, propiciando uma comunicação mais eficaz entre os membros das equipes multiprofissionais (Silva et al., 2017).

Portanto, faz-se necessário o desenvolvimento de uma cultura de segurança institucional orientada pelos princípios do planejamento, estratégias e avaliações contínuas. Destaca-se a importância da utilização da escala de checklist para o momento cirúrgico, elencando aspectos importantes para tornar o momento perioperatório mais seguro e provedor de uma assistência de enfermagem direcionada e holística (Silva et al., 2019).

No que tange ao uso desse checklist para a assistência em ambiente cirúrgico bem como para a segurança do paciente, essa ferramenta busca colaborar para superação de alguns obstáculos. Contudo, ressalta-se, como limitação do estudo, o uso de amostra não probabilística e a quantidade reduzida das instituições que participaram do estudo.

\section{Conclusão}

A validação pode-se dizer que o checklist encontra-se apropriado para utilização, compreendendo-o como uma ferramenta que agrega valores à segurança do paciente cirúrgico. Para tanto, percebeu-se que a padronização da contagem de material cirúrgico se torna relevante para a segurança do paciente cirúrgico e agrega valores fundamentais para uma política de saúde segura.

Assim, a importância da validação e aplicabilidade de um instrumento que subsidie na prevenção da retenção de itens na cavidade cirúrgica, traz implicações teóricas para a academia, como também, para uma prática segura nos centros cirúrgicos hospitalares. 
Deste modo, reforça-se a necessidade de novas pesquisas e a construção e aplicabilidade de ferramentas voltadas à prática de contagem de material cirúrgico de forma segura e efetiva.

\section{Referências}

Alexandre, N. M C. \& Coluci, M. O. (2011). Validade de conteúdo nos processos de construção e adaptação de instrumentos de medidas. Ciência \& Saúde Coletiva, 16(7), 3061-3068.

Alpendre, F. T., Cruz, E. D. A., Dyniewicz, A. M., Mantovani, M. F., Silva, A. E. B. C. \& Santos, G. S. (2017). Safe surgery: validation of pré and postoperative checklists. Revista Latino-Americana de Enfermagem, 25, 1-9.

Brasil. (2017). Práticas seguras para prevenção de retenção não intencional de objetos após realização de procedimento cirúrgico em serviços de saúde. Brasília, DF: ANVISA.

Brasil. (2012). Resolução n 466 do CNS, de 12 de dezembro de 2012. Aprova as diretrizes e normas regulamentadores de pesquisas envolvendo seres humanos. Brasília, DF: Conselho Nacional de Saúde.

Criado, J. V. N., Dias, B. F. \& Carmo, T. G. (2017). Processo de implementação do protocolo de cirurgia segura. Revista Cubana de Enfermería, 33 (1), 173189.

Fehring, R. J. (1994) The Fehring model. In R. M. Carrol-Johnson \& M. Paquete (Eds.), Classification of nursing diagnoses: proceedings of the tenth conference. Philadelphia: Lippincott Williams \& Wilkins.

Ferraz, E. M. (2009). A cirurgia segura: uma exigência do século XXI. Revista do Colégio Brasileiro de Cirurgiões, 36 (4), $281-282$.

Ferreira, N. C. S., Ribeiro, L., Mendonça, E. T. \& Amaro, M. O. F. (2019). Checklist de Cirurgia Segura: conhecimento e utilização do instrumento na perspectiva dos técnicos de enfermagem. Revista de Enfermagem do Centro-Oeste Mineiro, 16 (9), 1-8.

Freitas, M. R., Antunes, A. G., Azevedo, B. N. L., Fernandes, F. C., Monte, L. C. \& Gama, Z. A. S. (2014). Avaliação da adesão ao checklist de cirurgia segura da OMS em cirurgias urológicas e ginecológicas, em dois hospitais de ensino de Natal, Rio Grande do Norte, Brasil. Caderno de Saúde Pública, 30 (1), 137148.

Pasquali, L. (1998). Princípios de elaboração de escalas psicológicas. Revista de Psiquiatria Clínica, 25 (5), $206-213$.

Polit, D. F. \& Beck, C. T. (2011). Fundamentos de pesquisa em enfermagem: avaliação de evidências para as práticas da enfermagem. Artmed

Ribeiro, H. C. T. C., Quites, H. F. O., Bredes, A. C., Sousa, K. A. S. \& Alves, M. (2017). Adesão ao preenchimento do checklist de segurança cirúrgica. Cadernos de Saúde Pública, 33 (10), 1-13.

Sault Area Hospital. (2015). Surgical safety checklist compliance. https://sah.on.ca/patients/quality-safety/patient-safety-indicators/surgical-safety-checklistcompliance/

Sewell, M., Adebibe, M., Jayakumar, P., Jowett, C., Kong, K. \& Vemulapalli, K. (2011). Use of WHO surgical safety checklist in trauma and orthopaedic patients. International Orthopaedics, 35 (6), 897-901.

Silva, C. S. O., Pinheiro, G. O., Freitas, B. C., Figueiredo, B. M., Macedo, W. T. P. \& Silva, E. C. L. (2017). Checklist de cirurgia segura: os desafios da implantação e adesão nas instituições hospitalares brasileiras. Revista Espaço Ciência \& Saúde, 5 (2), 72-86.

Silva Júnior, S. D. \& Costa, F. J. (2014). Mensuração e escalas de verificação: uma análise comparativa das escalas de Likert e Phrase Completion. Revista Brasileira de Pesquisas de Marketing, Opinião e Mídia, 15 (3), 1-16. 